\title{
Decreasing $O$-GIcNAcylation affects the malignant transformation of MCF-7 cells via Hsp27 expression and its $\boldsymbol{O}$-GIcNAc modification
}

\author{
PUKKAVADEE NETSIRISAWAN ${ }^{1}$, PARUNYA CHAIYAWAT ${ }^{2,3}$, DARANEE CHOKCHAICHAMNANKIT ${ }^{1}$, \\ KRIENGSAK LIRDPRAPAMONGKOL ${ }^{1}$, CHANTRAGAN SRISOMSAP $^{1}$, \\ JISNUSON SVASTI $^{1,2}$ and VORARATT CHAMPATTANACHAI ${ }^{1,2}$ \\ ${ }^{1}$ Laboratory of Biochemistry, Chulabhorn Research Institute; ${ }^{2}$ Applied Biological Sciences Program, \\ Chulabhorn Graduate Institute, Bangkok 10210, Thailand
}

Received September 8, 2017; Accepted July 12, 2018

DOI: 10.3892/or.2018.6617

\begin{abstract}
O$-GlcNAcylation is a dynamic posttranslational modification of nucleoplasmic proteins. Previously, we reported that the $O$-GlcNAcylation level was increased in primary breast and colorectal cancer tissues. However, its precise roles in cancer development and progression are still largely unexplored. The aim of the present study was to investigate the roles of $O$-GlcNAcylation in the malignant transformation of cancer cell lines. $O$-GlcNAcylation level was examined in six cancer cell lines including breast (MCF-7 and MDA-MB-231), colorectal (SW480 and SW620), and liver (SK-Hep1 and HepG2). We found that the levels of $O$-GlcNAcylation and $O$-GlcNAc transferase (OGT), an $O$-GlcNAc catalyzing enzyme, were obviously increased in all cancerous cells, except SK-Hep1, when compared to normal cells. Reducing $O$-GlcNAcylation using RNA interference against OGT showed a marked reduction in OGT and $O$-GlcNAcylation levels. Surprisingly, siOGT had no effect on cell growth under conventional monolayer cultures. However, it inhibited anchorage-independent growth in soft agar cultures of all cancer cells, except SK-Hep1. Under anoikis resistance conditions performed by spheroid cultures, siOGT treatment decreased viability only in MCF-7, SW480, and SW620 cells. Among them, OGT knockdown in MCF-7 cells revealed a high inhibitory effect on colony and spheroid
\end{abstract}

Correspondence to: Dr Voraratt Champattanachai, Laboratory of Biochemistry, Chulabhorn Research Institute, 54 Kamphaeng Phet 6, Laksi, Bangkok 10210, Thailand

E-mail: voraratt@cri.or.th

Present address: ${ }^{3}$ Orthopedic Laboratory and Research Network (OLARN) Center, Department of Orthopedics, Faculty of Medicine, Chiang Mai University, Chiang Mai 50200, Thailand

Key words: anchorage-independent growth, anoikis resistance, heat shock protein $27, O$-GlcNAcylation, $O$-GlcNAc transferase cultures. Using two-dimensional gel electrophoresis and mass spectrometric analysis, heat shock protein 27 (Hsp27) was found to be the highest upregulated protein upon OGT knockdown. Immunoblots revealed that the Hsp27 protein level was increased but its $O$-GlcNAc modification level was decreased in siOGT-treated cells. These changes were associated with the inhibition of MCF-7 cell transformation. Notably, double knockdown of OGT and Hsp27 showed a reversal in the inhibitory effect on colony and spheroid cultures. Collectively, these results indicate that $O$-GlcNAcylation is required for anoikis resistance and anchorage-independent growth of MCF-7 cells. Blocking this glycosylation by OGT knockdown may regulate both Hsp27 protein expression and its $O$-GlcNAc modification levels. This alteration may play vital roles in malignant transformation.

\section{Introduction}

Cancer cells can invade and metastasize from primary sites through the blood and lymphatic system to other distant sites in the body. Metastatic cells must detach from the primary site and escape from normal defense mechanisms including cell cycle arrest and apoptosis. Anoikis is a form of apoptosis that is triggered when cells detach from their surrounding extracellular matrix (ECM), resulting in the loss of cell-cell communication and growth signals. Metastatic tumor cells; however, can survive under this harsh condition and this adaptive process is also known as anoikis resistance. In addition, cancer cells can grow in the absence of anchorage to the ECM and their neighboring cells, a process termed anchorage-independent growth. Anoikis resistance and anchorage-independence are therefore crucial steps in a series of changes that tumor cells undergo during malignant transformation. Extensive studies on anoikis resistance mechanisms have been performed in cancers to identify molecular targets for preventing metastasis (1-3). Moreover, anchorage-independent growth of cancer cells in vitro (colony forming capacity in soft agar media) has been used to predict the tumor phenotype, particularly with respect to the potential for metastasis in primary breast and lung tumors (4). 
Most cancer cells exhibit altered metabolism characterized by increased glucose uptake. This metabolic shift can alter glucose metabolism to produce enough energy and build the biomolecules needed by cancer cells. This includes the hexosamine biosynthesis pathway (HBP), a minor branch of the glycolytic pathway. The end product of the HBP is uridine diphosphate $N$-acetylglucosamine (UDP-GlcNAc), a sugar donor which is used for classical glycosylation in the endoplasmic reticulum and Golgi apparatus, as well as for $O$-GlcNAcylation in the cytoplasm, nucleus and mitochondria. Protein $O$-GlcNAcylation is a post-translational modification of serine or threonine residues of various nuclear-cytoplasmic and mitochondrial proteins (5). This glycosylation is not static but dynamically regulated by two key enzymes, $O$-GlcNAc transferase (OGT) (6) and $O$-GlcNAcase (OGA) (7), for addition and removal of $O$-GlcNAc from proteins, respectively. Growing evidence suggests that an aberrant $O$-GlcNAcylation level is associated with malignancy (8-10).

Previously, we reported that the $O$-GlcNAcylation level is increased in primary breast and colorectal cancer tissues $(11,12)$. Although an enhanced level of this modification is observed in most cancers, its roles in malignant transformation are still largely unexplored. In this study, the levels of $O$-GlcNAcylation and OGT were examined in six cancer cell lines including breast (MCF-7 and MDA-MB-231), colorectal (SW480 and SW620), and liver (SK-Hep1 and HepG2) and their normal cells. In addition, we investigated the effects of reducing $O$-GlcNAcylation using RNA interference against the $O G T$ gene in these cancer cell lines under different culture conditions including conventional monolayer, anchorage-independent growth of cancer cells in vitro, and anoikis resistance. Further studies were also performed to identify the proteins affected by $O$-GlcNAc reduction in anoikis resistance and the results are discussed.

\section{Materials and methods}

Cell cultures. Gibco ${ }^{\mathrm{TM}}$ Dulbecco's modified Eagle's medium (DMEM), RPMI-1640, penicillin-streptomycin solution, and L-glutamine were purchased from Thermo Fisher Scientific, Inc. (Waltham, MA, USA). Hyclone ${ }^{\mathrm{TM}}$ fetal bovine serum (FBS) was obtained from GE Healthcare Life Sciences (Logan, UT, USA). Human cancer cell lines including breast (MDA-MB-231 and MCF-7), colorectal (SW480 and SW620), and liver (HepG2 and SK-Hep-1) were purchased from the American Type Culture Collection (ATCC; Rockville, MD, USA). MDA-MB-231, MCF-7 and HepG2 cells were cultured in DMEM supplemented with 10\% FBS. SW480, SW620 and SK-Hep-1 cells were maintained in RPMI-1640 medium supplemented with $10 \%$ FBS. Human normal mammary epithelial cells (HMECs) and its medium (Mammary Epithelial Cell Growth Medium, MEGM) were purchased from Lonza (Walkersville, MD, USA) and cultured as recommended by the manufacturer. Human normal colon epithelial cells (CCD $841 \mathrm{CoN}$ ) and human normal liver epithelial cells (THLE-3), from ATCC, were provided by Dr Jutamaad Satayavivad, Chulabhorn Research Institute, Thailand. CCD $841 \mathrm{CoN}$ was cultured in DMEM supplemented with $10 \%$ FBS, and $1 \%$ L-glutamine while THLE-3 was cultured in DMEM supplemented with 10\% FBS and 25 mmol/1 HEPES
(Sigma-Aldrich; Merck KGaA, Darmstadt, Germany). All culture media were supplemented with $100 \mathrm{U} / \mathrm{ml}$ penicillin and $100 \mathrm{mg} / \mathrm{ml}$ streptomycin. The cells were maintained in humidified atmosphere of $95 \%$ air and $5 \% \mathrm{CO}_{2}$ at $37^{\circ} \mathrm{C}$.

Assessment of O-GlcNAc transferase (OGT) and O-GlcNAcylation levels. The levels of $O$-GlcNAc, OGT and $\beta$-actin were determined by immunoblotting using monoclonal mouse anti-human $O$-GlcNAc antibody, RL2 (ab2739, Abcam, Cambridge, MA, UK), monoclonal rabbit anti-human OGT antibody (O6264, Sigma-Aldrich; Merck KGaA) and monoclonal mouse anti-human $\beta$-actin antibody (mAb3700, Cell Signaling Technology, Beverly, MA, USA) as previously described (13). Briefly, cells were lysed in RIPA buffer containing $1 \%$ protease inhibitor cocktail (Sigma-Aldrich; Merck KGaA) and $20 \mu \mathrm{mol} / 1$ Thiamet-G (Sigma-Aldrich; Merck KGaA). Protein samples $(20 \mu \mathrm{g})$ were separated by $10 \%$ SDS-PAGE and transferred to PVDF membranes (Millipore, Bedford, MA, USA). Duplicate gels with equal amount protein loading were performed; one for $O$-GlcNAc modified proteins and another for OGT and $\beta$-actin. The membranes were probed with specific primary antibodies at 1:1,000. Immunoblots were developed with WesternBright ${ }^{\mathrm{TM}}$ ECL (Advansta, Menlo Park, CA, USA). The signals were captured and measured using an image analysis program (ImageQuant LAS4000; GE Healthcare, Marlborough, MA, USA). $\beta$-actin was used to compare protein loading of cell lysates.

$R N A$ interference. siRNA oligonucleotides of $O$-GlcNAc transferase (OGT) (sense, 5'-UAAUCAUUUCAAUAA CUGCUUCUGC-3' and antisense, 5'-GCAGAAGCAG UUAUUGAAAUGAUUA-3') and scramble negative control medium GC duplex were designed and purchased from Invitrogen; Thermo Fisher Scientific, Inc. For monolayer cultures, transfection of the siRNA oligos into cancer cells was carried out using Invitrogen ${ }^{\mathrm{TM}}$ Lipofectamine $2000^{\mathrm{TM}}$ in a forward transfection mode as described previously (11). For soft agar and anoikis resistance cultures, cells were transfected using a reverse transfection mode as described according to the manufacturer's instructions (Invitrogen; Thermo Fisher Scientific, Inc).

Monolayer culture and cell growth assay. Cancer cell growth was assessed by monitoring cell viability throughout 5 days using the MTT [3-(4,5-dimethylthiazol-2-yl)-2,5-diphenyltetrazolium bromide] assay in monolayer cultures as previously described (14). Briefly, cell suspensions were seeded into 96-well plates, cultured for 1 day, and transfected with RNA interference. Transfected cells were further cultured for 1-5 days. Then, the wells were replaced and incubated with fresh culture media containing $0.5 \mathrm{mg} / \mathrm{ml}$ MTT (SigmaAldrich; Merck KGaA) for $2 \mathrm{~h}$ at $37^{\circ} \mathrm{C}$. Finally, the medium was removed and replaced with DMSO (100 $\mu \mathrm{l} / \mathrm{well})$. Absorbance was measured at $550 \mathrm{~nm}$ and subtracted with the absorbance at $650 \mathrm{~nm}$, using a microplate reader. The number of viable cells was determined from the absorbance. Cultures with at least three independent wells were studied. The number of viable cells in each day was normalized by those of the Scramble siRNA at day 1, and reported as the percent relative cell growth. 
Anchorage-independent growth assay. Anchorage-independent cell growth assay in vitro was performed using soft agar cultures as described previously (11). Briefly, 1x104 cells (OGT knockdown and scramble cells using a reverse transfection mode) were suspended in $1 \mathrm{ml}$ top agar medium (the complete medium with $0.4 \%$ agar). The cell suspension was then overlaid onto $1.5 \mathrm{ml}$ bottom agar medium (the complete medium with $0.8 \%$ agar) in 6 -well culture plates in triplicate. Fresh complete medium was added to plates every 3 days as a feeder layer. Once colonies were propagated (MDA-MB231 and SW620 for 21 days, MCF-7, SK Hep-1 and HepG2 for 18 days, and SW480 for 28 days), they were stained with $0.005 \%$ crystal violet in $50 \%$ methanol for $1 \mathrm{~h}$, and images of the stained wells were captured. Colony number counts and average sizes were determined by ImageJ software version 1.42I (National Institutes of Health, Bethesda, MD, USA). All images were saved in an 8 bit format. The measured area was selected by elliptical selection and the threshold image was set using the threshold tool. The mode of analyzing particles was used with parameters of size: 1-infinity and circularity: 0.00-1.00. Cultures with at least two independent wells were studied. The results were reported as the average \pm standard deviation of colony number and size in OGT-knockdown cells normalized by those in the siScramble control.

Anoikis resistance assay. Anoikis resistance of cancer cells was determined by culturing the cells in polyHEMA-coated plates as descried previously with some modifications (2). Briefly, 2x10 5 cells (OGT-knockdown and scramble cells using a reverse transfection mode) were cultured in polyHEMAcoated plates. The polyHEMA-coated plates were prepared by soaking $30 \mathrm{mg} / \mathrm{ml}$ poly-HEMA (Sigma-Aldrich; Merck $\mathrm{KGaA}$ ) in $95 \%$ ethanol and putting this onto the plates and drying at $37^{\circ} \mathrm{C}$ in an incubator, followed by extensive washing with water and UV sterilization. After culturing for 3 days, cells were photographed using an inverted phase-contrast microscope at $\times 10$ magnification and harvested by centrifugation at $1,000 \mathrm{x} \mathrm{g}$ for $5 \mathrm{~min}$. After harvesting, the cell pellets were resuspended in PBS buffer, and incubated with trypsinEDTA solution (Gibco; Thermo Fisher Scientific, Inc.) for $10 \mathrm{~min}$. The dissociated cells were collected for measurement of OGT and $O$-GlcNAc levels as described above. Aliquots of the collected cells were further determined for viability of anoikis-resistant cells using trypan blue dye exclusion assay. Trypsinized cells $(20 \mu \mathrm{l})$ were mixed with $20 \mu \mathrm{l}$ of $0.04 \%$ trypan blue. Viable and non-viable cells from four microscopic viewing areas were counted using a hemocytometer. Cultures with at least two independent wells were studied. The results were reported as the average \pm standard deviation of anoikisresistant cells (viable cells) in the OGT-knockdown cells normalized by those in the siScramble control.

Two-dimensional gel electrophoresis (2-DE). 2-DE was performed to measure the global protein alteration between OGT-knockdown and scramble control groups. Briefly, cultured cells $\left(2 \times 10^{6}\right)$ were lysed with 2-D lysis buffer, $1 \%$ protease inhibitor cocktail (Sigma-Aldrich; Merck KGaA), homogenized and harvested as described previously (11). Protein samples $(100 \mu \mathrm{g})$ were electro-separated by isoelectric focusing (IEF) in immobilized $\mathrm{pH}$ gradient (IPG) strips,
pH 3.0-10.0, followed by 10\% SDS-PAGE. After electrophoresis was performed, proteins on the gels were stained using $0.1 \%$ Coomassie brilliant blue R-250 (CBB). Protein spots were scanned using ImageScanner (Amersham Biosciences; GE Healthcare, Chicago, IL, USA) and quantitatively measured by ImageMaster 2-DE Platinum 7.0 software (GE Healthcare). Three independent experiments were performed. The relative intensity of each protein spot showing statistically significant difference was selected and reported as a fold change between two groups (OGT knockdown vs. Scramble control).

Protein identification by mass spectrometric analysis. Protein spots with statistical difference in expression levels were excised from the gel, destained and enzymatically digested by trypsin (Promega, Madison, WI, USA). The digested peptides were then identified using Nanoflow liquid chromatography coupled with the amaZon speed ion trap mass spectrometry (Bruker, Billerica, MA, USA) as previously described (13). MASCOT search with NCBInr version 20130630 sequence databases (http://www.matrixscience.com) was performed in order to identify the protein spots. Search parameters were set as follows: peptide mass tolerance was 1.2 Da, MS/MS ion mass tolerance was 0.6 Da, allowance was set to 1 missed cleavage, enzyme set as trypsin, the limit of peptide charges was $1+, 2+$ and $3+$. Decoy was marked. Proteins with molecular weight (MW) and $\mathrm{pI}$ consistent to the spot on 2-DE gel and total ion scores $>80$ units ( $\mathrm{P}$-value $<0.05$ ) were considered positively identified.

Confirmation of Hsp27 protein and O-GlcNAc-Hsp27 levels. The expression level of Hsp27 in the siOGT and siScramble cells was confirmed by immunoblotting (IB). Protein samples $(20 \mu \mathrm{g})$ were separated by $10 \%$ SDS-PAGE, transferred onto PVDF membranes, and probed using monoclonal mouse antihuman Hsp27 antibody (1:2,000; cat. no. ab2790; Abcam, Cambridge, MA, USA). Immunoblots were developed with WesternBright ECL and the signals were captured as described above. $\beta$-actin was used to compare protein loading of cell lysates. The results were reported as the average band intensity \pm standard deviation of Hsp27 in the OGT-knockdown cells normalized by those in the siScramble control. At least three independent experiments were performed.

Hsp27 was further confirmed to be $O$-GlcNAc modified using immunoprecipitation (IP). Briefly, proteins $(1,000 \mu \mathrm{g})$ in low-salt lysis buffer were incubated with antibodies against Hsp27 (1:250; cat. no. ab2790; Abcam) and RL2 (1:200; cat. no. ab2739; Abcam). The suspensions were mixed gently by shaking in an end-over-end manner at $4^{\circ} \mathrm{C}$ for overnight. After that, the immune complexes were incubated with Protein A and $\mathrm{G}$ Sepharose (GE Healthcare) for $2 \mathrm{~h}$ to perform coupling reaction. After the washing steps, the immune complexes were eluted by adding $25 \mu \mathrm{l}$ of $2 \mathrm{X}$ sampling buffer and heating at $95-100^{\circ} \mathrm{C}$ for $10 \mathrm{~min}$. The eluted samples were loaded onto 12.5\% SDS-PAGE and immunoblotted with RL2 and Hsp27 antibodies to determine the levels of $O$-GlcNAc-Hsp27.

Double knockdown of OGT and Hsp27. siRNA-mediated gene silencing against OGT (Invitrogen; Thermo Fisher Scientific, Inc.) and Hsp27 (sc-29350, Santa Cruz Biotechnology, Dallas, TX, USA) was performed in MCF-7 cells. Transfections 
A

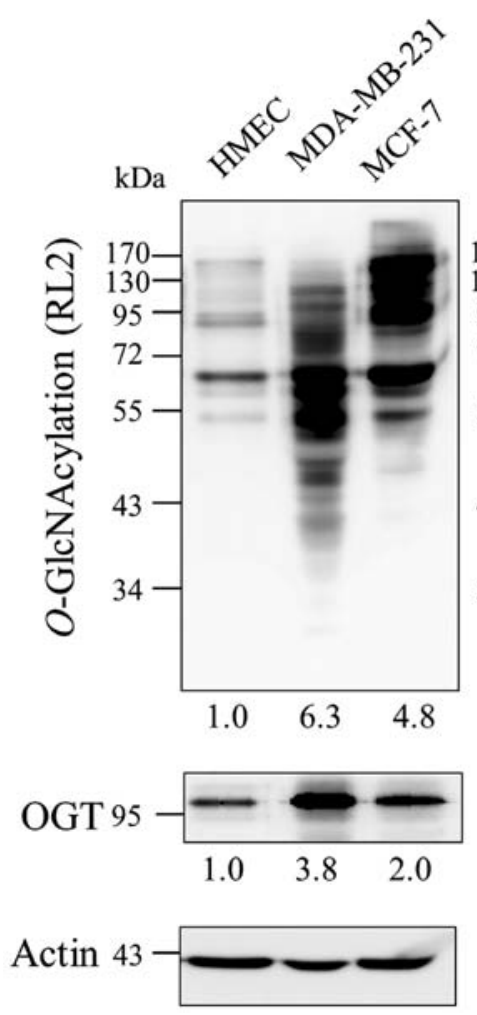

B

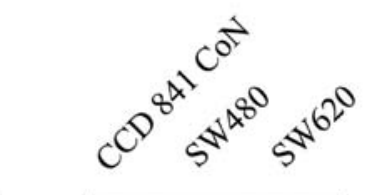

C
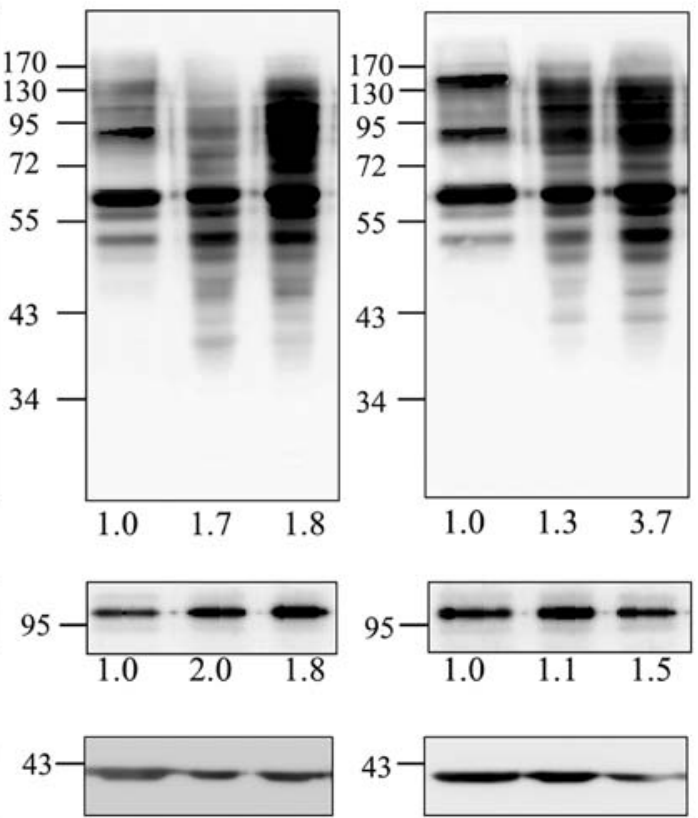
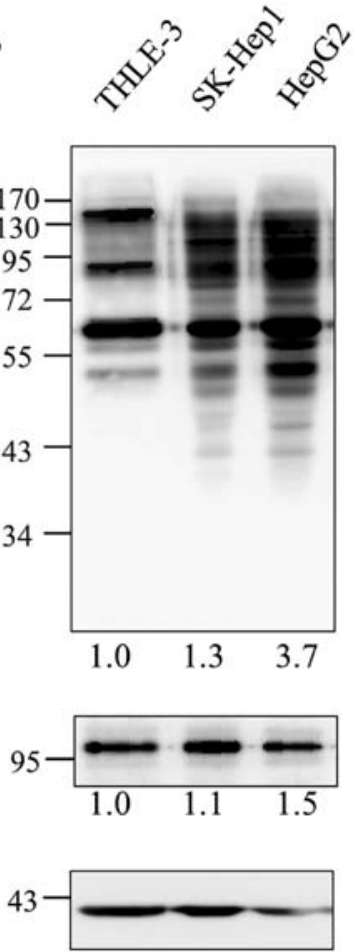

Figure 1. $O$-GlcNAcylation and OGT levels in normal and cancer cells of the breast, colon and liver. The levels of $O$-GlcNAcylation and OGT in cell lysates of (A) breast (HMEC, MDA-MB-231 and MCF-7), (B) colon (CCD841 CoN, SW480 and SW620) and (C) liver (THLE-3, SK-Hep1 and HepG2) cell lines were determined using $O$-GlcNAcylation (RL2) and OGT immunoblotting. $\beta$-actin immunoblotting was performed as an internal control for the intensity normalization of RL2 and OGT levels in each cell line. Values provided under the immunoblots are the relative protein intensities normalized by those in the normal cells (HMEC for breast, CCD841 CoN for colon, and THLE-3 for liver). The data show one representation of two independent experiments.

were performed in reverse transfection mode using Lipofectamine $2000^{\mathrm{TM}}$ as described above. The effects of siRNA of Hsp27, OGT and Hsp27/OGT on anchorage-independent cell growth and anoikis-resistant cells were examined using soft agar cultures and trypan blue dye exclusion assay, respectively, as described above.

Statistical analysis. The statistical analysis was conducted using unpaired Student's t-test to test for the difference between two groups. One-way ANOVA was used where appropriate followed by a Bonferroni's multiple comparison test using Prism 5.0 of GraphPad Software Inc. (GraphPad Software, Inc., San Diego CA, USA). The statistical significance was defined as $\mathrm{P}<0.05$ and $\mathrm{P}<0.01$.

\section{Results}

Augmentation of O-GlcNAcylation and O-GlcNAc transferase in cancer cells. Since several reports revealed that the $O$-GlcNAcylation level is increased in many types of cancer, we examined the levels of this modification and its catalyzing enzyme, OGT in cancer cell lines with different invasive capability, in comparison to their normal cells including breast (MCF-7 and MDA-MB-231 vs HMEC), colon (SW480 and SW620 vs CCD841 CoN) and liver (SK-Hep1 and HepG2 vs THLE-3). $O$-GlcNAcylation and OGT levels were increased in all tested cancer cell lines ( $\geq 1.5$ fold), except SK-Hep1 in which its OGT level was not different but many more $O$-GlcNAc protein bands were obviously observed when compared to those of THLE-3 (Fig. 1). Of note, both $O$-GlcNAc modification and OGT levels in breast cancer cell lines appeared to be higher than those in colon and liver cancer cell lines.

Transient knockdown of O-GlcNAc transferase in conventional monolayer cultures. Since $O$-GlcNAylation and OGT levels were upregulated in the cancer cell lines, transient knockdown of $O$-GlcNAylation by RNA interference against OGT was performed in six cancer cell lines. siOGT treatment of six cancer cell lines showed a marked reduction of both OGT and $O$-GlcNAcylation levels in comparison with the siScramble control group (Fig. 2A). Indeed, we observed that siOGT suppressed the OGT and $O$-GlcNAcylation levels in our culture system by more than $70 \%$. However, surprisingly, OGT knockdown had little or no effect on cell viability and growth, even though experiments were performed up to 5 days of transfection (Fig. 2B). At some time points, fluctuation of cell viability was observed in siOGT treatment, but this was not consistent in a time-dependent manner.

Transient knockdown of O-GlcNAc transferase in soft agar cultures. Culturing cells in soft agar is based on the colony formation in anchorage-independent growth, which is considered the most accurate and stringent in vitro assay for detecting malignant transformation of cells. Since OGT knockdown had 
A
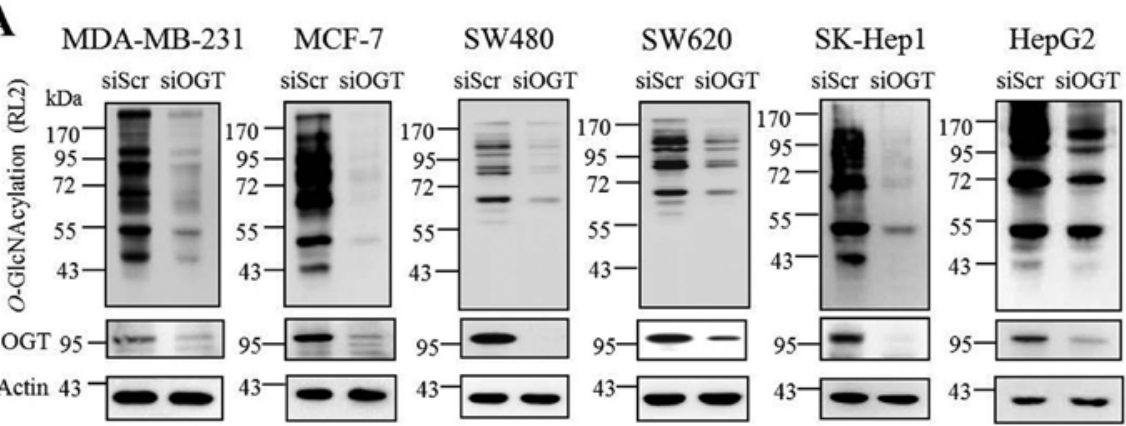

B
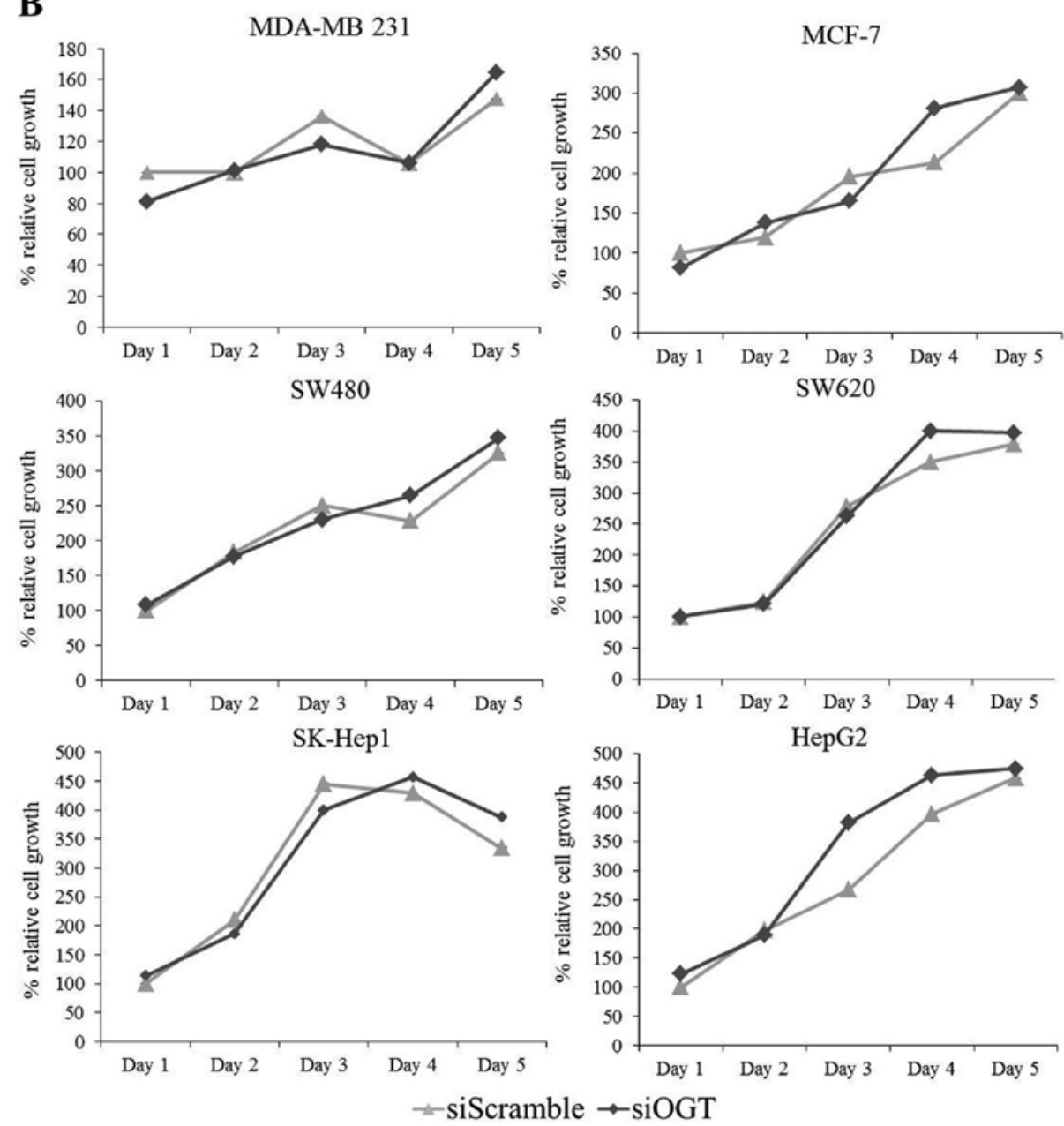

Figure 2. OGT knockdown and viability of breast, colon and liver cancer cells cultured in conventional monolayer cultures. siRNA transfection against OGT (siOGT) or scramble (siScr)was performed in MDA-MB-231, MCF-7, SW480, SW620, SK-Hep1 and HepG2 cells for 1-5 days. (A) Representative immunoblotting of $O$-GlcNAcylation (RL2), OGT and $\beta$-actin in the cell lysates of six cancer cell lines at Day 3 of RNAi transfection. (B) Effect of siOGT and siScramble treatments on the cell growth of six cancer cell lines for a 5-day interval culturing. Data are presented as the average \pm SD from three independent experiments.

no effect on the cell viability and growth in the monolayer culture, we aimed to ascertain whether the reduction of this modification may affect anchorage-independent growth in vitro. Anchorage-independent cell growth assay of siOGT and siScramble cells of six cancer cell lines were performed using soft agar cultures as shown in Fig. 3A. Notably, all cancer cell lines treated with siOGT treatment, except SK-Hep1, displayed a significant reduction in colony number (Fig. 3B) and colony size (Fig. 3C) when compared to those of the siScramble cell groups, respectively. Surprisingly, OGT knockdown was unlikely to affect colony formations in SK-Hep1 cells.
Transient knockdown of O-GlcNAc transferase in anoikis resistance cultures. Cancer cells can resist anoikis and thereby survive after detachment from their primary site and can travel through the circulatory systems as circulating cancer cells. In this study, anoikis resistance was assessed in vitro by culturing cells on polyHEMA-coated plates. As shown in Fig. 4A, all cancer cells were able to grow as floating spheroids in the medium culture. Among the six cancer cell lines, OGT knockdown statistically decreased viable cells only in the MCF-7, SW480 and SW620 cell lines while it exhibited a weaker effect on MDA-MB-231 and HepG2 cells and no effect was observed in the SK-Hep1 cells (Fig. 4B). We also confirmed 


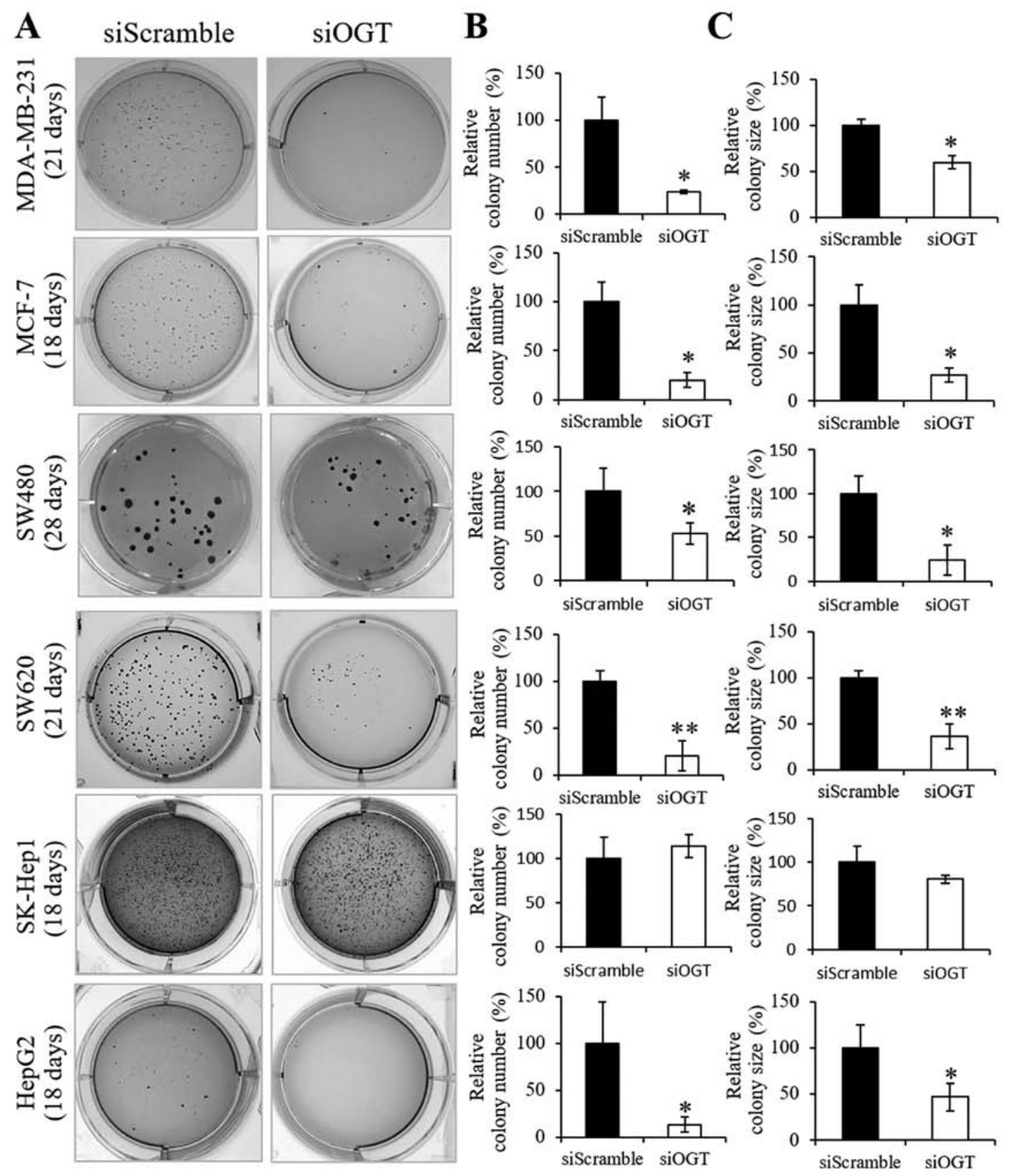

Figure 3. OGT knockdown and viability of breast, colon and liver cancer cells cultured in soft agar cultures. siRNA transfection against OGT (siOGT) or scramble (siScramble) was performed in MDA-MB-231, MCF-7, SW480, SW620, SK-Hep1 and HepG2 cells and cultured for the indicated time. (A) Representative colony formation in soft agar stained by crystal violet. (B and C) Graphs represent relative colony numbers and sizes in the siOGT and siScramble groups. Data are presented as the average \pm SD from three independent experiments. ${ }^{*} \mathrm{P}<0.05$ and ${ }^{* *} \mathrm{P}<0.01$.

that, under the anoikis resistance culture conditions, OGT and $O$-GlcNAcylation levels were still reduced following siOGT treatment when compared to levels in the siScramble control group (Fig. 4C).

Alteration of protein expression in anoikis-resistant cells by OGT knockdown. Since siOGT treatment caused a statistically significant decrease in anoikis resistance of MCF-7 cells, two-dimensional (2-DE) gel electrophoresis and mass spectrometric analysis were used to determine which proteins would be affected when the OGT level was reduced under anoikisresistant conditions. Image analysis revealed that 7 protein spots were differentially expressed in the siOGT-transfected cells compared to those in the siScramble control group ( $\geq 1.5$ fold, $\mathrm{P}<0.05$ ) (Fig. 5). Then, these 7 upregulated proteins were identified by mass spectrometric analysis and the results are shown in Table I. These identified proteins were categorized into 3 groups based on their protein functions. The first was heat shock protein 27 (Hsp27) (spot no. 1 and no. 2) which is involved in chaperone/stress response. The second group was involved in energy metabolism including triosephosphate isomerase (TPI) (spot no. 3), inorganic pyrophosphatase (spot no. 5), PCTP-like protein (spot no. 6) and nucleoside diphosphate kinase A (spot no. 7). The last protein was peroxiredoxin-2 (spot no. 4) which is involved in cellular protection/ detoxification. 
A
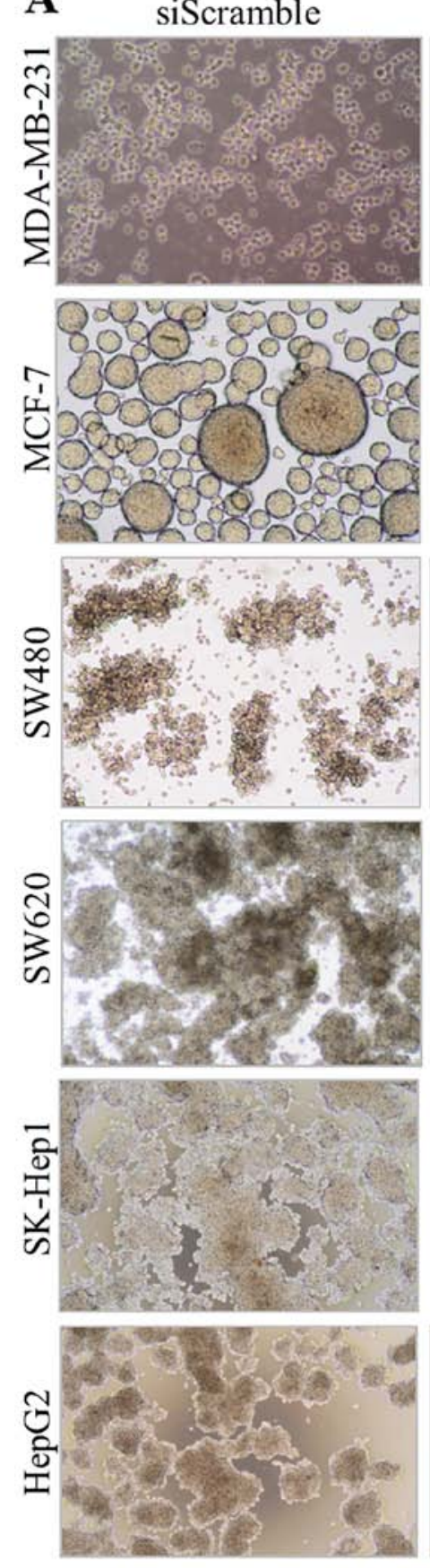

siOGT
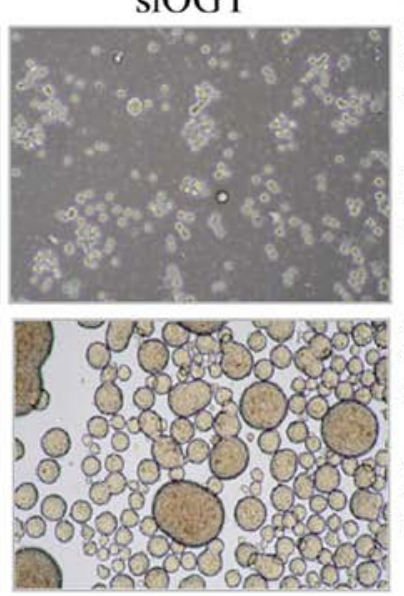

B
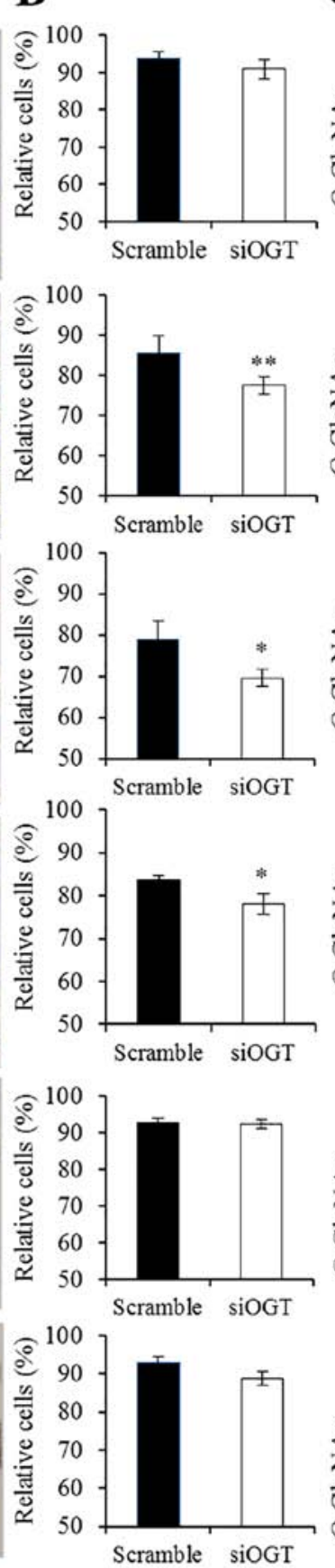

C
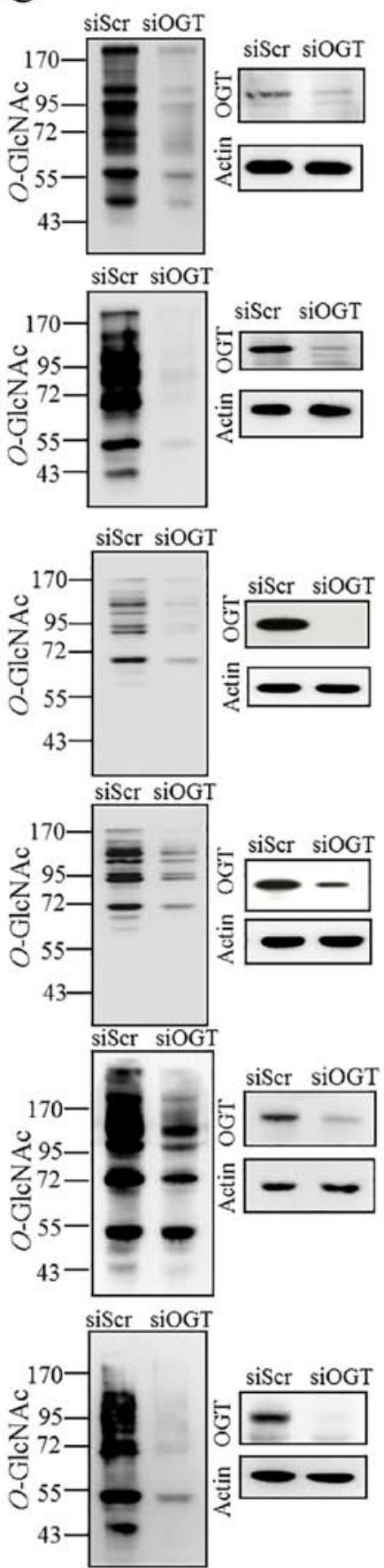

Figure 4. OGT knockdown and viability of breast, colon and liver cancer cells cultured in polyHEMA-coated plates. siRNA transfection against OGT (siOGT) or scramble (siScramble) was performed in MDA-MB-231, MCF-7, SW480, SW620, SK-Hep1 and HepG2 cells and cultured for 3 days. (A) Images represent cells cultured on polyHEMA-coated plates taken with x10 magnification. (B) Graphs represent the relative percentage of anoikis-resistant cells (viable cells) between the siOGT and siScramble groups. Data are presented as the average \pm SD from three independent experiments. (C) Representative immunoblotting of O-GlcNAc modified proteins (RL2), OGT and $\beta$-actin in cell lysates from the siOGT and siScramble groups. ${ }^{*} \mathrm{P}<0.05$ and ${ }^{* *} \mathrm{P}<0.01$.

Reduction of $O$-GlcNAcylation and Hsp27 expression and its $O$-GlcNAc modification. As determined by 2-DE and mass spectrometric analysis, the expression level of Hsp27 was the most markedly increased upon OGT knockdown. Therefore, Hsp27 which exerts chaperone/stress response functions was selected for further validation by immunoblotting. The results showed that the expression level of Hsp27 was increased by siOGT knockdown in comparison to this level in the siScramble control with significantly higher relative band intensity $(\mathrm{P}<0.01)$ (Fig. 6A). Using Hsp27 immunoprecipitation and immunoblot analysis of Hsp27 and $O$-GlcNAc (RL2) antibodies, we found that Hsp27 was modified by $O$-GlcNAc (Fig. 6B and C). The level of $O$-GlcNAc-modified Hsp27 was obviously decreased following siOGT transfection when compared to the level in the siScramble control. The reduction in the $O$-GlcNAc-modified Hsp27 level following siOGT may be the result of a global decrease in the $O$-GlcNAcylation level upon OGT knockdown. 

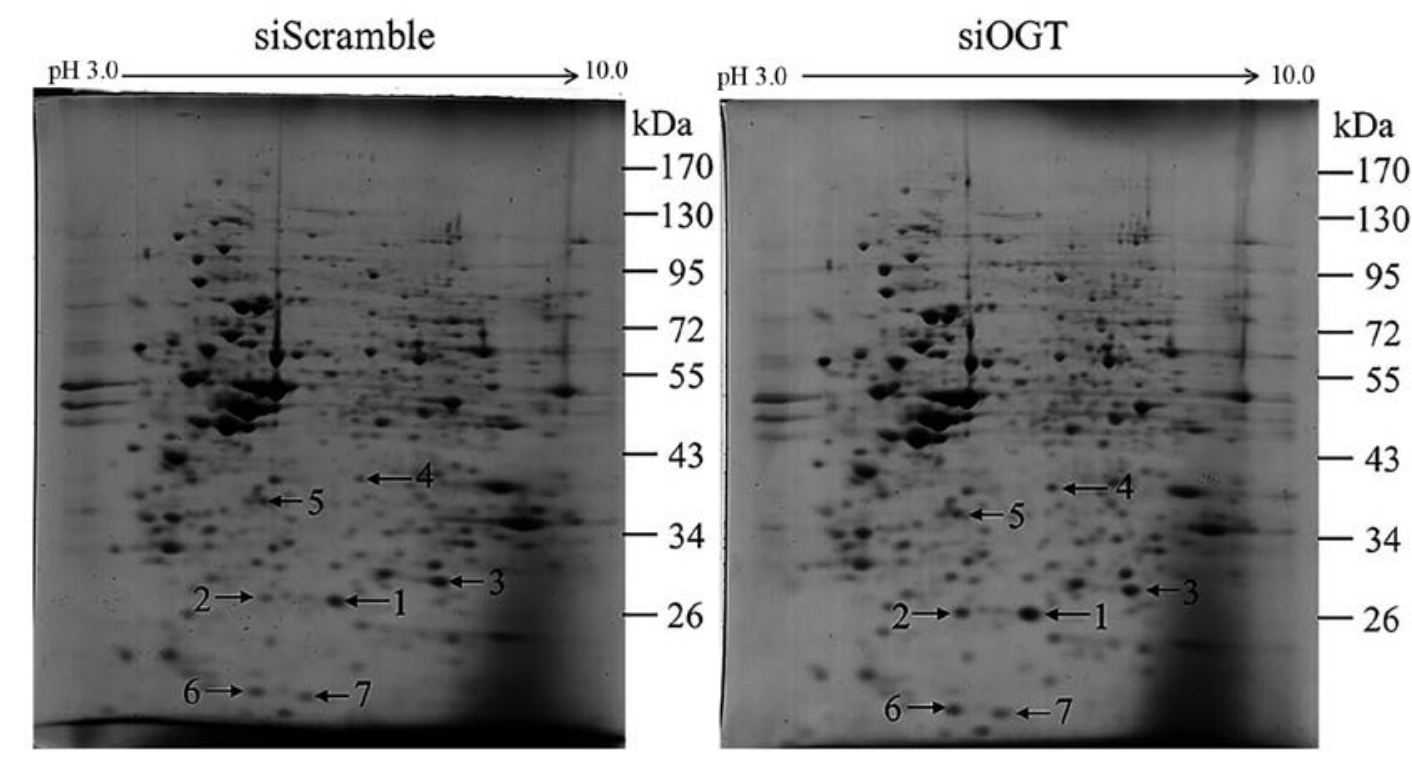

Figure 5. Representative two-dimensional (2-DE) protein stained gels of siScramble and siOGT-knockdown MCF-7 cells. Proteins (100 $\mu \mathrm{g})$ from the siOGTand siScramble-transfected cells cultured under anoikis resistance for 3 days were separated by IEF (pH 3.0-10.0) followed by 10\% SDS-PAGE and stained by Coomassie blue R-250. Arrows with numbers indicate protein spots which were differentially expressed $\geq 1.5$ fold between the two groups and further identified by mass spectrometric analysis including (spot no. 1) and (no. 2) heat shock protein 27, (no. 3) triosephosphate isomerase, (no. 4) peroxiredoxin-2, (no. 5) inorganic pyrophosphatase, (no. 6) PCTP-like protein and (no. 7) nucleoside diphosphate kinase A. The data show one representation of three independent experiments.

Table I. List of upregulated proteins found in siOGT-transfected compared to siScramble-treated MCF-7 cells culturing under aniokis resistance conditions.

\begin{tabular}{|c|c|c|c|c|c|c|c|c|}
\hline No. & Protein name & Database & $\begin{array}{l}\text { Accession } \\
\text { number }\end{array}$ & $\begin{array}{c}\mathrm{MW} / \mathrm{pI} \\
(\mathrm{kDa} / \mathrm{pI})\end{array}$ & $\begin{array}{l}\text { Peptide } \\
\text { matches }\end{array}$ & Score & $\begin{array}{l}\text { Fold-change } \\
\text { (siOGT/siScr) }\end{array}$ & P-value \\
\hline 1 & Heat shock protein 27 & NCBInr & gil4504517 & $22.768 / 5.98$ & 8 & 398 & 2.22 & $<0.001$ \\
\hline 2 & Heat shock protein 27 & NCBInr & gil4504517 & $22.768 / 5.98$ & 5 & 207 & 2.0122 & 0.012 \\
\hline 3 & Triosephosphate isomerase & NCBInr & gil136066 & $26.894 / 7.10$ & 8 & 394 & 1.66 & 0.04 \\
\hline 4 & Peroxiredoxin-2 & NCBInr & gi:32189392 & $22.049 / 5.66$ & 3 & 89 & 1.55 & 0.04 \\
\hline 5 & Inorganic pyrophosphatase & NCBInr & gil11056044 & $33.095 / 5.54$ & 2 & 92 & 1.51 & 0.04 \\
\hline 6 & PCTP-like protein & NCBInr & gil116812600 & $33.427 / 6.67$ & 3 & 103 & 1.5 & 0.05 \\
\hline 7 & Nucleoside diphosphate kinase A & NCBInr & gil35068 & $17.138 / 5.83$ & 4 & 148 & 1.5 & 0.008 \\
\hline
\end{tabular}

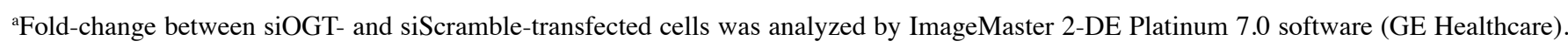
MW, molecular weight, pI, isoelectric point.

Double knockdown of OGT and Hsp27. According to previous results, it has been suggested that the elevation of Hsp27 may be associated with the suppression of anoikis resistance and anchorage-dependent growth of MCF-7 cells. Therefore, we determine whether the reduction in Hsp27 is capable to regain the growth in anoikis-resistant cultures under an OGT silencing condition. Transient knockdown of Hsp27 was performed to diminish the Hsp27 expression level. Hsp27 immunoblotting revealed a decreased level of Hsp27 in siHsp27 and siOGT/siHsp27 transfected cells whereas $O$-GlcNAc immunoblotting showed that the $O$-GlcNAc level was reduced in the siOGT and siOGT/siHsp27 cells when compared to those of siScramble controls (Fig. 7A). Double knockdown of siOGT/siHsp27 (siDouble) reversed the inhibitory effect in anoikis-resistant cultures compared to that of the siOGT-transected cells (Fig. 7B). Moreover, double knockdown of siOGT/siHsp27 markedly restored the growth of MCF-7 cells in soft agar cultures when compared to that of the siOGT-transected alone cells (Fig. 7C).

\section{Discussion}

Emerging evidence reveals that aberrant glycosylation is associated with pathobiological states of various diseases including cancer. In general, cancer cells require a high uptake of glucose for their rapid growth. Some glucose can enter into the hexosamine biosynthesis pathway (HBP), a minor branch of glycosis, which is responsible for producing a sugar donor, UDP-GlcNAc, for glycosylation reactions including $O$-GlcNAcylation. Increases in the HBP flux, UDP-GlcNAc 
A

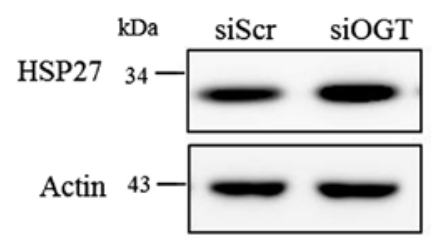

B Crude $\frac{\text { Hsp27 IP }}{\text { Neg. siScr siOGT }}$

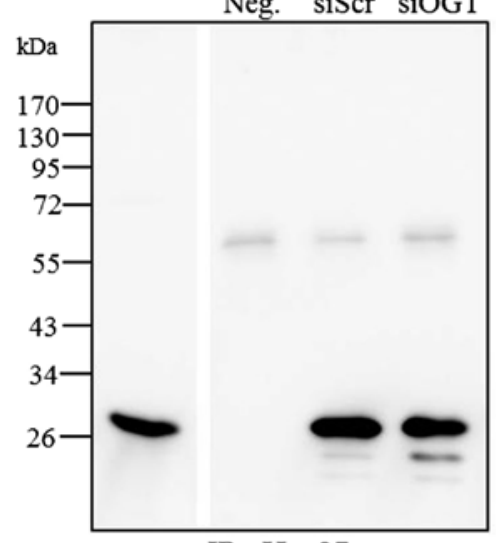

IP : Hsp27

IB : Hsp27

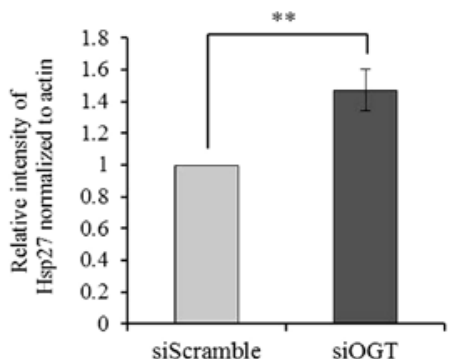

Crude

Hsp27 IP

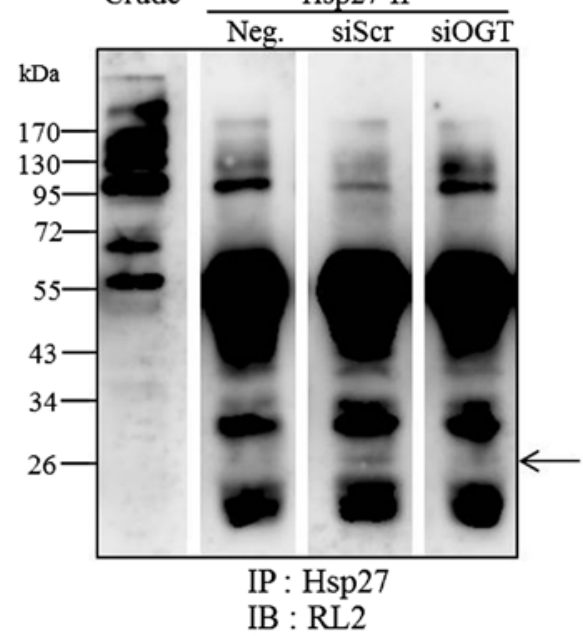

IB : RL2

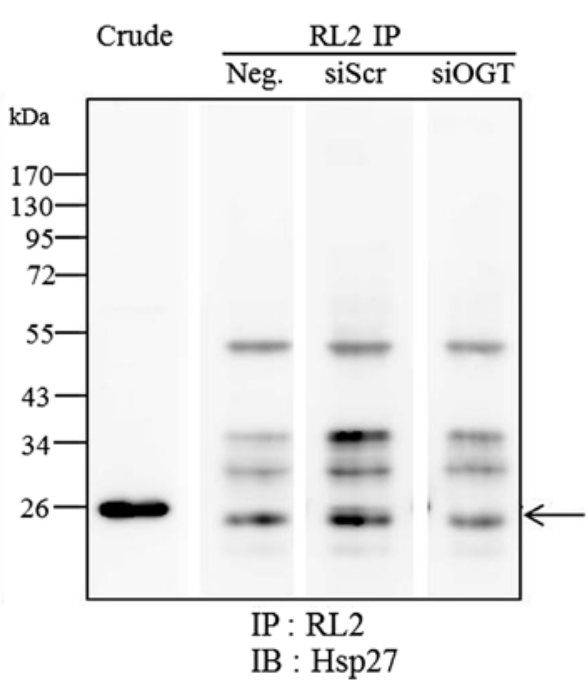

Figure 6. Confirmation of Hsp27 alteration and $O$-GlcNAc-Hsp27 levels under anoikis resistance. (A) Representative immunoblot for Hsp27 and $\beta$-actin (protein loading control) and graph represents the relative intensity of Hsp27 normalized by $\beta$-actin. The data show one representation of four independent experiments. ${ }^{* *} \mathrm{P}<0.01$. (B) Immunoblots of Hsp27 and $O$-GlcNAc (RL2) with Hsp27 IP. (C) Immunoblots of RL2 and Hsp27 with RL2 IP. Direct IP was performed in siRNA against OGT (siOGT), scramble (siScr) and negative control (Neg.). Arrows indicate $O$-GlcNAc-Hsp27.

and $O$-GlcNAcylation, are therefore directly related to the high glucose uptake generally observed in malignant cells. In this study, we examined the $O$-GlcNAcylation level in human cancer cell lines originating from the breast (MDA-MB-231 and MCF-7), colon (SW480 and SW620) and liver (SK-Hep1 and HepG2) cells. Most cancer cell lines, except SK-Hep1, showed an increased modification level in comparison to that of their normal cell counterparts. This increase was also consistent with the upregulation of OGT expression level (Fig. 1). Less change in $O$-GlcNAc and OGT levels, on the other hand, were observed in SK-Hepl cells. This indicates that the regulation of $O$-GlcNAcylation and its controlling enzymes in various cancer cell lines may be different. However, previous research from our laboratory and others reported that augmentation of $O$-GlcNAcylation and OGT levels were associated with the malignant phenotypes of most cancers including breast $(11,15)$, colon $(12,16,17)$, liver $(18-20)$ and prostate $(21,22)$. It is noted that the regulation of $O$-GlcNAcylation is dynamic and may not be only dependent on the expression levels of its cycling enzymes (OGT and OGA) but also on its enzymatic activities (23). Further investigation, therefore; is needed to clarify what other factors regulate this dynamic modification. 
A

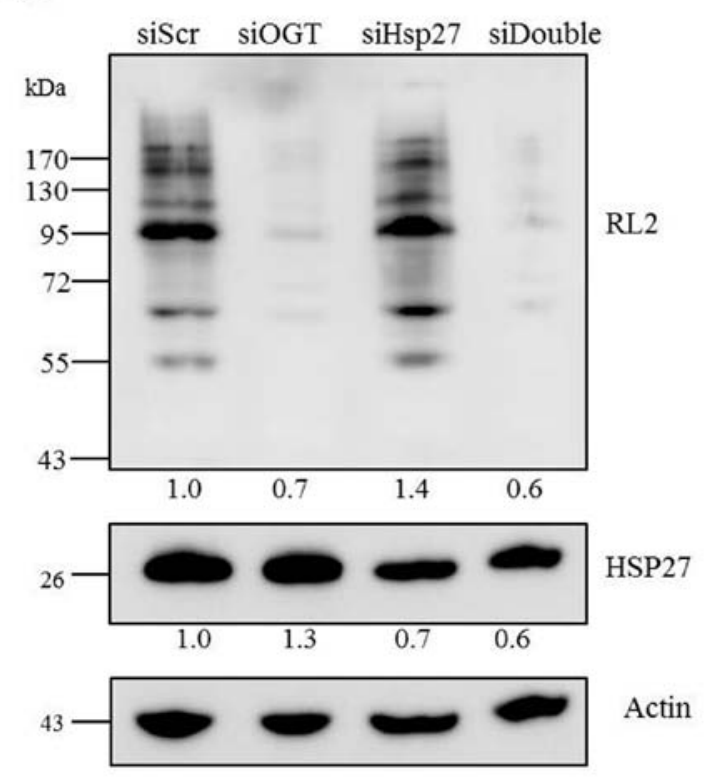

B

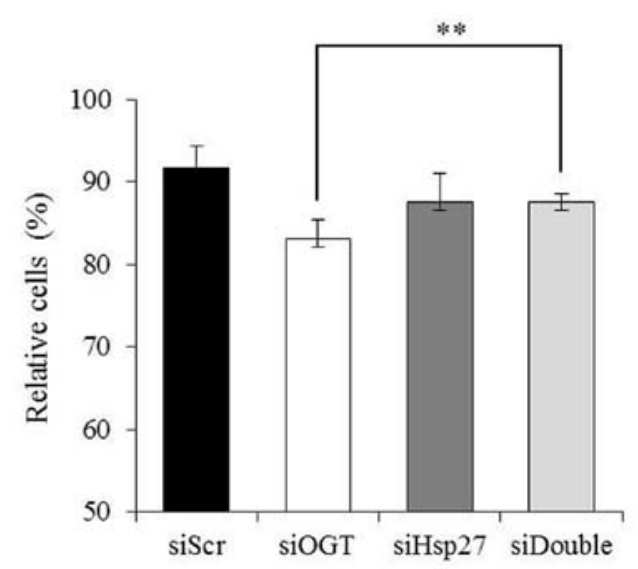

C

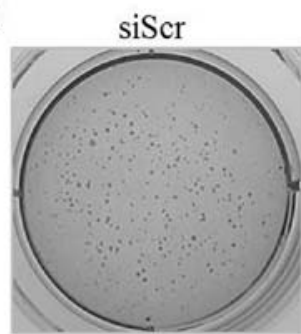

siHsp27

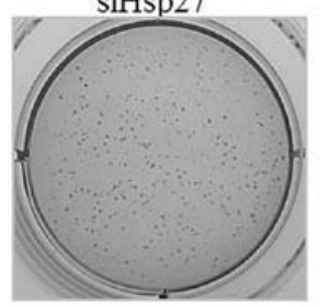

siOGT

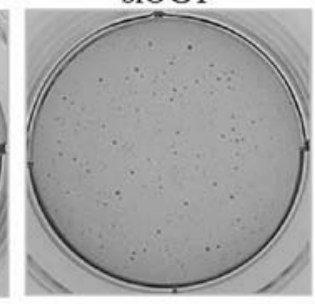

siDouble

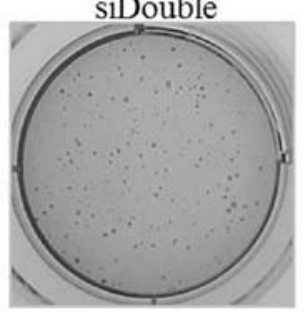

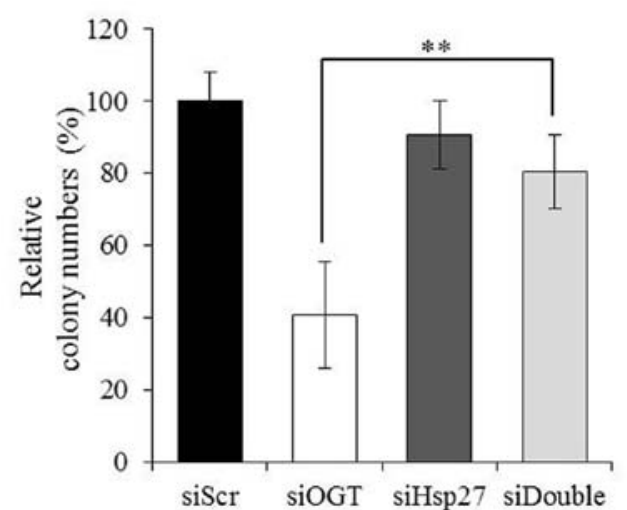

Figure 7. Effects of double knockdown of OGT and Hsp27 in polyHEMA-coated plates and soft agar cultures of MCF-7 cells. Double knockdown of OGT and Hsp27 (siDouble), single knockdown of OGT (siOGT), single knockdown of Hsp27 (siHsp27) and scramble (siScr) were performed in MCF-7 cells. (A) Immunoblotting of Hsp27, O-GlcNAc modified proteins (RL2) and $\beta$-actin at Day 3 of RNA silencing in polyHEMA-coated plate cultures. Values provided under the immunoblots are the relative protein intensities normalized by $\beta$-actin. (B) Graph represents the relative percentage of anoikis-resistant cells (viable cells) of MCF-7 cells in siDouble, siOGT, siHsp27 and siScr at Day 3 of RNA silencing in polyHEMA-plate cultures. (C) Representative colony formation stained by crystal violet and the graph represents the relative colony number of siDouble, siOGT, siHsp27 and siScr at Day 18 of RNA silencing in soft agar cultures. Data are presented as the average $\pm \mathrm{SD}$ from three independent experiments. ${ }^{* *} \mathrm{P}<0.01$.

OGT is vital for cellular survival. Deletion of OGT results in embryonic lethality (24). Moreover, reduction in OGT, together with stress stimuli treatment, caused a dramatic decrease in cell viability (25). Surprisingly, reduction in $O$-GlcNAcylation through RNA interference of OGT did not appear to alter cell growth and proliferation under conventional monolayer cultures, but instead inhibited colony formation of breast cancer cells under anchorage-independent growth $(15,26)$. From these findings, we aimed to ascertain whether OGT may be required for anchorage-independent growth in other cancer cell types. Interestingly, the results showed that reducing the OGT level could not affect the growth in monolayer cultures of the tested cancer cell lines (Fig. 2). In contrast, siOGT treatment caused a decrease in colony formation, in terms of both numbers and sizes, compared to those of the siScramble controls, except for SK-Hepl cells (Fig. 3). Consistent with this result, other groups also reported that decreased $O$-GlcNAcylation caused a reduction in colony formation, as observed by others in lung (17), prostate (22) and pancreatic cancers (27). In addition, six cancer cell lines were also cultured on polyHEMA-coated plates to determine the anoikis resistance of the cancer cells. Under this culture condition, reduction in the $O$-GlcNAcylation level affected anoikis-resistant growth of lowly invasive adenocarcinoma (MCF-7, SW480 and SW620), but had weaker effects on highly invasive adenocarcinoma cells (MDA-MB-231 and SK-Hep1) and even liver cells (HepG2) (Fig. 4). According to our results, OGT knockdown had a high inhibitory effect on colony and spheroid formations of MCF-7 cells whereas it had 
little or no effect on SK-Hepl cells. This indicates some correlation between cell type and the anoikis-resistant property. Moreover, the mechanisms underlying anchorage-independent growth and anoikis resistance may differ, thus the role of $O$-GlcNAcylation may depend on both cellular properties and cellular adaptation to tumor microenvironmental changes.

OGT may regulate $O$-GlcNAcylation and protein levels of target proteins. In this study, since siOGT treatment strongly affected both anchorage-independent growth and anoikis resistance of MCF-7 cells, we examined total protein expression levels in anoikis-resistant cells to determine those affected by OGT reduction. Results from 2-DE images and mass spectrometric analysis demonstrated that at least 7 proteins were upregulated in the MCF-7 cells with decreased OGT (Fig. 5 and Table I). These upregulated proteins are involved in many cellular processes including chaperone/stress response (Hsp27), cellular metabolism (PCTP-like protein, inorganic pyrophosphatase, triosephosphate isomerase (TPI), and nucleoside diphosphate kinase A), and protection/detoxification (peroxiredoxin-2). Previously, we demonstrated that $O$-GlcNAcylation and OGT levels were increased in primary breast malignant tumors and the $O$-GlcNAc modification levels of 29 proteins including TPI were altered in breast cancer tissues when compared to those of adjacent tissues (11). In parallel, Chaiyawat et al reported that, in colon cancer cell lines, pyruvate kinase M2 (PKM2) was $O$-GlcNAcylated and its protein level was increased while its $O$-GlcNAc level was decreased upon OGT knockdown (13). In the present study, the TPI level was upregulated upon OGT knockdown. In addition to TPI, we found that the Hsp27 level was increased but its $O$-GlcNAc level was decreased in siOGT cells when compared to levels in the siScramble control cells. From these changes observed in PKM2, TPI and Hsp27, it is possible that OGT knockdown not only reduced the $O$-GlcNAc modification of target proteins but also affected the level of target proteins by controlling at transcription/translation levels. Further investigation is needed to clarify how $O$-GlcNAcylation regulates protein expression levels.

Hsp27 is a chaperone of the small heat shock protein group. We found that the Hsp27 protein level was most highly increased upon OGT knockdown under anoikis resistance conditions (Figs. 5 and 6). Hsp27 was reported to be implicated in various cellular processes or even under pathologic disease conditions including cancer (28). Its expression level affects cell proliferation, migration and invasion in many types of cancer i.e., liver, prostate and breast cancer (29-31). Generally, Hsp27 is involved in the stress response mechanism which restores protein homeostasis (adaptive mechanism) in cancer cells. It is therefore possible that a decreased global $O$-GlcNAcylation level (by siOGT knockdown) may induce harsh conditions resulting in an accumulation of misfolded proteins and/or other stress-responsive factors, as shown by the upregulation of Hsp27 in this study. In contrast, a number of reports suggest that Hsp27 can serve as a tumor suppressor which acts against cancer progression and metastasis. For instance, overexpression of Hsp27 is sufficient to inhibit pulmonary fibrosis and lung tumorigenesis by diminishing endothelial-to-mesenchymal transition (EndMT) (32). Additionally, increased expression of Hsp27 could efficiently suppress lung metastasis of colorectal cancer in vivo (33).
Hsp27 expression in salivary gland tumor tissues has been reported to be higher in benign tumors than in malignant tumors (34). As demonstrated in both soft agar and spheroid conditions (Fig. 7), double knockdown of Hsp27 and OGT led to a decrease in the Hsp27 level, resulting in the reversal of this inhibitory effect of MCF-7 growth. An increased Hsp27 level upon OGT knockdown, therefore, was likely to be causal for inhibiting cancer cell formation. However, how increased Hsp27 is able to suppress the malignancy of MCF-7 cells is an unsolved mystery worthy of further investigation.

The gene/protein regulation of Hsp27 is complicated. Hsp27 expression level is regulated by specificity protein 1 (Sp1) (35), which is an ubiquitous transcription factor that can activate or repress transcription of many genes in response to physiological and pathological stimuli. Sp1 is reported to be $O$-GlcNAc-modified and overexpression of OGT is shown to inhibit Sp1 transcriptional activity (36). Therefore, reduction of $O$-GlcNAcylation by OGT knockdown may increase Sp1 activity and Hsp27 expression levels. Guo et al reported that Hsp27 is modified by $O$-GlcNAc (37). They suggested that increased $O$-GlcNAc modification of Hsp27 enhanced the translocation of Hsp27 from the cytoplasm into the nucleus and this may be a novel regulatory state of Hsp27 functions. In our study, we found that Hsp27 was $O$-GlcNAc modified (Fig. 6). Although the overall level of Hsp27 was increased, the $O$-GlcNAc-Hsp27 level was decreased upon OGT knockdown in anoikis resistance conditions (Fig. 6). Further studies, therefore; are needed to clarify whether OGT knockdown causes less Hsp27 entry into the nucleus.

In summary, an increase in the $O$-GlcNAcylation level modulated by high glucose uptake and overexpression of OGT may be an important malignant phenotype observed in most cancers. Many researchers are trying to block or reduce the action of OGT using inhibitors and/or knockdown of the $O G T$ gene so as to inhibit cancer progression and development. However, the precise roles of $O$-GlcNAcylation in cancer development and progression are still elusive. Anoikis resistance and anchorage-independent growth are vital steps for metastatic tumors. Our data suggest that, in MCF-7 cells, $O$-GlcNAcylation is required for both processes, and blocking this glycosylation by OGT knockdown affected these processes, at least in part, via the regulation of Hsp27 expression and its $O$-GlcNAc modification. This information may further elucidate the potential mechanism of $O$-GlcNAc modification associated with cancer progression, especially in breast cancer. Nevertheless, further studies are required to determine this precise mechanism.

\section{Acknowledgements}

The authors would like to thank Dr Jutamaad Satayavivad, Chulabhorn Research Institute, Thailand, for culturing of human normal colon epithelial cells (CCD $841 \mathrm{CoN})$ and human normal liver epithelial cells (THLE-3).

\section{Funding}

The present study was supported by the Thailand Research Fund (grant no. TRG5580006), the Chulabhorn Research Institute and the Chulabhorn Graduate Institute, Thailand. 


\section{Availability of data and materials}

The datasets used during the present study are available from the corresponding author upon reasonable request.

\section{Authors' contributions}

PN and VC conceived and designed the study. PN and PC performed the experiments in cell cultures, gel proteomics and immunoblotting. DC performed the LC-MS/MS. KL and CS interpreted the results and reviewed the manuscript. JS reviewed and edited the manuscript and was involved in the conception of the study. PN and VC wrote and drafted the manuscript. All authors read and approved the manuscript and agree to be accountable for all aspects of the research in ensuring that the accuracy or integrity of any part of the work are appropriately investigated and resolved.

\section{Ethics approval and consent to participate}

Not applicable.

\section{Patient consent for publication}

Not applicable.

\section{Competing interests}

The authors declare that they have no competing interests.

\section{References}

1. Howard EW, Leung SC, Yuen HF, Chua CW, Lee DT, Chan KW, Wang $X$ and Wong YC: Decreased adhesiveness, resistance to anoikis and suppression of GRP94 are integral to the survival of circulating tumor cells in prostate cancer. Clin Exp Metastasis 25: 497-508, 2008.

2. Khongmanee A, Lirdprapamongkol K, Tit-oon P, Chokchaichamnankit D, Svasti J and Srisomsap C: Proteomic analysis reveals important role of 14-3-3 $\sigma$ in anoikis resistance of cholangiocarcinoma cells. Proteomics 13: 3157-3166, 2013.

3. Kim JB, Yu JH, Ko E, Lee KW, Song AK, Park SY, Shin I, Han W and Noh DY: The alkaloid Berberine inhibits the growth of Anoikis-resistant MCF-7 and MDA-MB-231 breast cancer cell lines by inducing cell cycle arrest. Phytomedicine 17: 436-440, 2010.

4. Mori S, Chang JT, Andrechek ER, Matsumura N, Baba T, Yao G, Kim JW, Gatza M, Murphy S and Nevins JR: Anchorageindependent cell growth signature identifies tumors with metastatic potential. Oncogene 28: 2796-2805, 2009.

5. Hart GW, Housley MP and Slawson C: Cycling of O-linked beta-N-acetylglucosamine on nucleocytoplasmic proteins. Nature 446: 1017-1022, 2007.

6. Kreppel LK, Blomberg MA and Hart GW: Dynamic glycosylation of nuclear and cytosolic proteins. Cloning and characterization of a unique $O$-GlcNAc transferase with multiple tetratricopeptide repeats. J Biol Chem 272: 9308-9315, 1997.

7. Gao Y, Wells L, Comer FI, Parker GJ and Hart GW: Dynamic $\mathrm{O}$-glycosylation of nuclear and cytosolic proteins: Cloning and characterization of a neutral, cytosolic beta-N-acetylglucosaminidase from human brain. J Biol Chem 276: 9838-9845, 2001.

8. Ma Z and Vosseller K: $O$-GlcNAc in cancer biology. Amino Acids 45: 719-733, 2013.

9. Fardini Y,Dehennaut V,Lefebvre T and Issad T: $O$-GlcNAcylation: A New Cancer Hallmark? Front Endocrinol (Lausanne) 4: 99 , 2013.

10. Chaiyawat $P$, Netsirisawan $P$, Svasti J and Champattanachai V: Aberrant $O$-GlcNAcylated proteins: New perspectives in breast and colorectal cancer. Front Endocrinol (Lausanne) 5: 193, 2014.
11. Champattanachai V, Netsirisawan $P$, Chaiyawat $P$, Phueaouan T, Charoenwattanasatien R, Chokchaichamnankit D, Punyarit P, Srisomsap C and Svasti J: Proteomic analysis and abrogated expression of $O$-GlcNAcylated proteins associated with primary breast cancer. Proteomics 13: 2088-2099, 2013.

12. Phueaouan T, ChaiyawatP,Netsirisawan P,ChokchaichamnankitD, Punyarit P, Srisomsap C, Svasti J and Champattanachai V: Aberrant $O$-GlcNAc-modified proteins expressed in primary colorectal cancer. Oncol Rep 30: 2929-2936, 2013.

13. Chaiyawat P, Chokchaichamnankit D, Lirdprapamongkol K, Srisomsap C, Svasti J and Champattanachai V: Alteration of $O$-GlcNAcylation affects serine phosphorylation and regulates gene expression and activity of pyruvate kinase M2 in colorectal cancer cells. Oncol Rep 34: 1933-1942, 2015.

14. Chiablaem K, Lirdprapamongkol K, Keeratichamroen S, Surarit R and Svasti J: Curcumin suppresses vasculogenic mimicry capacity of hepatocellular carcinoma cells through STAT3 and PI3K/AKT inhibition. Anticancer Res 34: 1857-1864, 2014.

15. Caldwell SA, Jackson SR, Shahriari KS, Lynch TP, Sethi G, Walker S, Vosseller K and Reginato MJ: Nutrient sensor $O$-GlcNAc transferase regulates breast cancer tumorigenesis through targeting of the oncogenic transcription factor FoxM1. Oncogene 29: 2831-2842, 2010.

16. Steenackers A, Olivier-Van Stichelen S, Baldini SF, Dehennaut V, Toillon RA, Le Bourhis X, El Yazidi-Belkoura I and Lefebvre T: Silencing the nucleocytoplasmic $O$-GlcNAc transferase reduces proliferation, adhesion, and migration of cancer and fetal human colon cell lines. Front Endocrinol (Lausanne) 7: 46, 2016.

17. Mi W, Gu Y, Han C, Liu H, Fan Q, Zhang X, Cong Q and Yu W: $O$-GlcNAcylation is a novel regulator of lung and colon cancer malignancy. Biochim Biophys Acta 1812: 514-519, 2011.

18. Zhang X, Qiao Y, Wu Q, Chen Y, Zou S, Liu X, Zhu G, Zhao Y, Chen Y, Yu Y, et al: The essential role of YAP $O$-GlcNAcylation in high-glucose-stimulated liver tumorigenesis. Nat Commun 8: $15280,2017$.

19. Liu Q, Tao T, Liu F, Ni R, Lu C and Shen A: Hyper- $O$ GlcNAcylation of YB-1 affects Ser102 phosphorylation and promotes cell proliferation in hepatocellular carcinoma. Exp Cell Res 349: 230-238, 2016.

20. Zhu G, Tao T, Zhang D, Liu X, Qiu H, Han L, Xu Z, Xiao Y, Cheng $\mathrm{C}$ and Shen A: $O$-GlcNAcylation of histone deacetylases 1 in hepatocellular carcinoma promotes cancer progression. Glycobiology 26: 820-833, 2016.

21. Itkonen HM, Gorad SS, Duveau DY, Martin SE, Barkovskaya A, Bathen TF, Moestue SA and Mills IG: Inhibition of $O$-GlcNAc transferase activity reprograms prostate cancer cell metabolism. Oncotarget 7: 12464-12476, 2016.

22. Gu Y, Gao J, Han C, Zhang X, Liu H, Ma L, Sun X and Yu W: $O$-GlcNAcylation is increased in prostate cancer tissues and enhances malignancy of prostate cancer cells. Mol Med Rep 10: 897-904, 2014.

23. Yang X and Qian K: Protein $O$-GlcNAcylation: Emerging mechanisms and functions. Nat Rev Mol Cell Biol 18: 452-465, 2017.

24. O'Donnell N, Zachara NE, Hart GW and Marth JD: Ogt-dependent X-chromosome-linked protein glycosylation is a requisite modification in somatic cell function and embryo viability. Mol Cell Biol 24: 1680-1690, 2004.

25. Zachara NE, O'Donnell N, Cheung WD, Mercer JJ, Marth JD and Hart GW: Dynamic $O$-GlcNAc modification of nucleocytoplasmic proteins in response to stress. A survival response of mammalian cells. J Biol Chem 279: 30133-30142, 2004.

26. Gu Y, Mi W, Ge Y, Liu H, Fan Q, Han C, Yang J, Han F, Lu X and $\mathrm{Yu}$ W: GlcNAcylation plays an essential role in breast cancer metastasis. Cancer Res 70: 6344-6351, 2010.

27. Ma Z, Vocadlo DJ and Vosseller K: Hyper- $O$-GlcNAcylation is anti-apoptotic and maintains constitutive NF- $\kappa B$ activity in pancreatic cancer cells. J Biol Chem 288: 15121-15130, 2013.

28. Arrigo AP and Gibert B: HspB1, HspB5 and HspB4 in human cancers: Potent oncogenic role of some of their client proteins. Cancers (Basel) 6: 333-365, 2014.

29. Hung CS, Huang CY, Lee CH, Chen WY, Huang MT, Wei PL and Chang YJ: IGFBP2 plays an important role in heat shock protein 27-mediated cancer progression and metastasis. Oncotarget 8: 54978-54992, 2017.

30. Cordonnier T, Bishop JL, Shiota M, Nip KM, Thaper D, Vahid S, Heroux D, Gleave M and Zoubeidi A: Hsp27 regulates $\mathrm{EGF} / \beta$-catenin mediated epithelial to mesenchymal transition in prostate cancer. Int J Cancer 136: E496-E507, 2015. 
31. Gibert B, Eckel B, Gonin V, Goldschneider D, Fombonne J, Deux B, Mehlen P, Arrigo AP, Clézardin P and Diaz-Latoud C: Targeting heat shock protein 27 (HspB1) interferes with bone metastasis and tumour formation in vivo. Br J Cancer 107: 63-70, 2012.

32. Choi SH, Nam JK, Kim BY, Jang J, Jin YB, Lee HJ, Park S, Ji YH, Cho J and Lee YJ: HSPB1 inhibits the endothelial-tomesenchymal transition to suppress pulmonary fibrosis and lung tumorigenesis. Cancer Res 76: 1019-1030, 2016.

33. Lee YJ, Lee HJ, Choi SH, Jin YB, An HJ, Kang JH, Yoon SS and Lee YS: Soluble HSPB1 regulates VEGF-mediated angiogenesis through their direct interaction. Angiogenesis 15: 229-242, 2012.

34. Wang G, Gu X, Chen L, Wang Y, Cao B and E Q: Comparison of the expression of 5 heat shock proteins in benign and malignant salivary gland tumor tissues. Oncol Lett 5: 1363-1369, 2013.
35. Mo XM, Li L, Zhu P, Dai YJ, Zhao TT, Liao LY, Chen GG and Liu ZM: Up-regulation of Hsp27 by ER $\alpha / S p 1$ facilitates proliferation and confers resistance to apoptosis in human papillary thyroid cancer cells. Mol Cell Endocrinol 431: 71-87, 2016.

36. Yang X, Su K, Roos MD, Chang Q, Paterson AJ and Kudlow JE: $\mathrm{O}$-linkage of $\mathrm{N}$-acetylglucosamine to $\mathrm{Sp} 1$ activation domain inhibits its transcriptional capability. Proc Natl Acad Sci USA 98: 6611-6616, 2001.

37. Guo K, Gan L, Zhang S, Cui FJ, Cun W, Li Y, Kang NX, Gao MD and Liu KY: Translocation of HSP27 into liver cancer cell nucleus may be associated with phosphorylation and $O$-GlcNAc glycosylation. Oncol Rep 28: 494-500, 2012. 